\title{
Desenvolvimento e validação de um instrumento para avaliar intervenções em relação aos princípios da Promoção da Saúde
}

doi: 10.1590/\$1679-49742021000300005

\author{
Development and validation of an instrument to evaluate interventions in relation to Health \\ Promotion principles
}

Desarrollo y validación de un instrumento para evaluar intervenciones con relación a los principios de la Promoción de la Salud

\author{
Mathias Roberto Loch ${ }^{1}$ - (1) orcid.org/0000-0002-2680-4686 \\ Emmanuelly Correia de Lemos ${ }^{2}$ - (1) orcid.org/0000-0003-1450-6160 \\ Patrícia Constante Jaime ${ }^{3}$ - (1) orcid.org/0000-0003-2291-8536 \\ Cassiano Ricardo Rech ${ }^{4}$ - (1) orcid.org/0000-0002-9647-3448 \\ 'Universidade Estadual de Londrina, Programa de Pós-Graduação em Saúde Coletiva, Londrina, PR, Brasil \\ ${ }^{2}$ Secretaria Estadual de Saúde de Pernambuco, Recife, PE, Brasil \\ ${ }^{3}$ Universidade de São Paulo, Faculdade de Saúde Pública, São Paulo, SP, Brasil \\ ${ }^{4}$ Universidade Federal de Santa Catarina, Programa de Pós-Graduação em Educação Física, Florianópolis, SC, Brasil
}

\section{Resumo}

Objetivo: Desenvolver e validar um instrumento destinado a avaliar a proximidade de intervenções que buscam promover saúde, em relação aos princípios da Política Nacional de Promoção da Saúde do Brasil. Métodos: Estudo transversal de desenvolvimento e validação de um instrumento, a partir de consulta a 20 trabalhadores, na análise da primeira versão, 19 na versão final, e mais 31 trabalhadores na análise da fidedignidade e da avaliação de conteúdo, realizada por dez especialistas na versão inicial e 12 na versão final. Foram utilizados o índice de validade de conteúdo, o alpha de Cronbach $(\alpha)$ e o coeficiente de correlação intraclasse. Resultados: A adequação dos indicadores foi de 89,8\%; a clareza de 82,5\%; a consistência interna de $\alpha=0,80$, e a correlação teste-reteste, de 0,93. Conclusão: 0 instrumento apresentou validade e fidedignidade aceitáveis, podendo ser utilizado na avaliação de intervenções que tenham o objetivo de promover a saúde.

Palavras-chave: Promoção da Saúde; Estudos de Validação; Inquéritos e Questionários; Modelos de Assistência à Saúde.

\section{Endereço para correspondência:}

Mathias Roberto Loch - Universidade Estadual de Londrina, Departamento de Educação Física, Campus Universitário, Rodovia Celso Garcia Cid, PR 445, km 380, Londrina, PR, Brasil. CEP: 86057-970

E-mail: mathiasuel@hotmail.com 


\section{Introdução}

Promoção da Saúde é uma expressão que guarda muitos conceitos, associada a valores e princípios diversos e amplos, como qualidade de vida, solidariedade, democracia, cidadania, desenvolvimento, participação social e intersetorialidade, entre outros. ${ }^{1-9}$ No Brasil, um marco importante para a institucionalização da Promoção da Saúde foi a implantação da Política Nacional de Promoção da Saúde (PNPS), publicada em 2006 e reformulada em 2014. ${ }^{10-12}$ A PNPS tem, como objetivo principal,

promover a equidade e a melhoria das condições e dos modos de viver, ampliando a potencialidade da saúde individual e coletiva e reduzindo vulnerabilidades e riscos à saúde decorrentes dos determinantes sociais, econômicos, políticos, culturais e ambientais. ${ }^{10}$

E pretende, ademais, desencadear mudanças nos modos de organizar, planejar, realizar, analisar e avaliar 0 trabalho em saúde. ${ }^{13}$

\section{A criação de instrumentos que auxiliem a avaliação das intervenções destinadas a promover saúde pode ser de grande relevância para se avaliar a menor ou maior proximidade de cada intervenção com os princípios da Promoção da Saúde.}

Os diversos atores envolvidos nas intervenções que buscam promover saúde precisam considerar e buscar promover certos princípios. A criação de instrumentos que auxiliem a avaliação das intervenções destinadas a promover saúde pode ser de grande relevância, inclusive para se avaliar a menor ou maior proximidade de cada intervenção com os princípios da Promoção da Saúde. Portanto, é preciso garantir 0 adequado processo de desenvolvimento e validação desses instrumentos na população-alvo. ${ }^{14}$

0 objetivo deste estudo foi desenvolver e validar um instrumento destinado a avaliar a proximidade de intervenções que buscam promover saúde, em relação aos princípios da PNPS do Brasil. Do público-alvo da aplicação do instrumento, formado pelos trabalhadores que atuam em intervenções cujo foco é a Promoção da Saúde, espera-se a avaliação desses programas, a análise - por eles próprios - de suas intervenções e sua proximidade com os princípios da PNPS. E, considerando-se as especificidades de cada intervenção e contexto, que adéquem seus programas de ação para estarem em sintonia com esses mesmos princípios.

\section{Métodos}

Realizou-se um estudo observacional, com delineamento descritivo e transversal. 0 contexto de aplicação do estudo foi a Atenção Primária à Saúde (APS) no Brasil, caracterizada por profissionais e residentes da área da saúde que atuam diretamente na APS, em ações de Promoção da Saúde. Para garantir maior representatividade de opinião dos profissionais, optou-se por incluir diferentes categorias desses trabalhadores, distribuídas entre diferentes regiões e cidades do país. Utilizou-se, como referencial metodológico, a psicometria. Trata-se de uma ciência voltada à medição de fenômenos não observáveis diretamente, embora possíveis de serem caracterizados utilizando-se outros atributos aferíveis. ${ }^{14}$

A seleção da amostra foi intencional, para garantir a participação de especialistas no conteúdo do instrumento a ser elaborado. 0 tamanho amostral foi definido de acordo com indicações da literatura. ${ }^{14}$ A Figura 1 apresenta as etapas metodológicas adotadas no estudo.

\section{Etapa 1}

\section{Estruturação da base teórica da escala e elaboração dos itens do instrumento}

Nesta etapa, buscou-se identificar os princípios da Promoção da Saúde e elaborar seus respectivos indicadores.

Para identificar esses princípios, foram utilizadas a PNPS $^{10} \mathrm{e}$ as Cartas das Conferências Internacionais de Promoção da Saúde. ${ }^{15-22}$ Uma primeira versão do instrumento foi desenhada, contendo 19 indicadores baseados em 16 princípios: (i) Equidade; (ii) Participação Social; (iii) Autonomia; (iv) Empoderamento; (v) Intrassetorialidade; (vi) Intersetorialidade; (vii) Sustentabilidade; (viii) Integralidade; (ix) Territorialidade; (x) Educação Popular em Saúde; (xi) Abrangência das Orientações; (xii) Reorientação dos Serviços de Saúde; (xiii) Cultura da Paz e Direitos Humanos; (xiv) Educação Permanente; (xv) Criação de Ambientes Favoráveis; e (xvi) Abordagem Positiva. 
Etapa 1

Estruturação da base teórica do instrumento e elaboração dos itens.

Etapa 2

Aplicação, com público-alvo (trabalhadores que atuam em intervenções em saúde), da versão inicial do instrumento.

\section{Etapa 3}

Validação de Conteúdo - Análise do instrumento por especialistas da área de Promoção da Saúde quanto à adequação e clareza dos princípios e dos respectivos indicadores da versão inicial do instrumento.

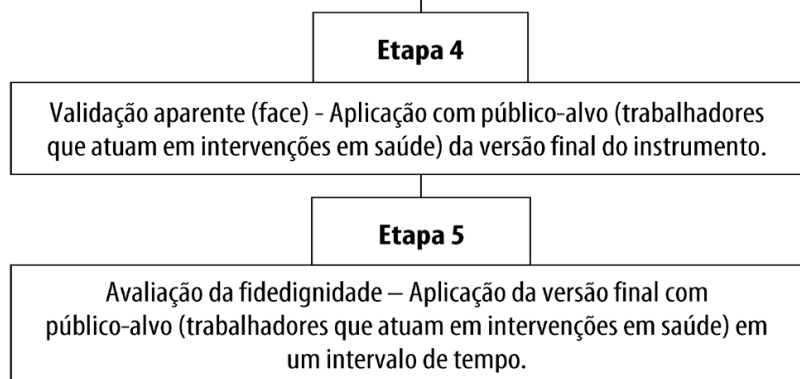

\section{Figura 1 - Etapas do desenvolvimento, validação de conteúdo e aparente (face), e fidedignidade de um instrumento para avaliar o nível de proximidade de intervenções em saúde com os princípios da Promoção da Saúde}

\section{Etapa 2}

\section{Aplicação junto ao público-alvo (trabalhadores que atuam em intervenções em saúde) da versão inicial do instrumento}

Nesta etapa, o objetivo foi verificar a compreensão do público-alvo da ideia central do instrumento e suas impressões quanto a sua aplicabilidade, antes da validação de conteúdo (etapa posterior). 0 instrumento inicial foi aplicado em uma turma de segundo ano da Residência em Saúde da Família da Universidade Estadual de Londrina - dez residentes de oito diferentes áreas da Saúde (Enfermagem, Educação Física, Farmácia, Fisioterapia, Nutrição, Odontologia, Psicologia e Serviço Social, sendo dois com formação inicial em enfermagem, dois em odontologia e um de cada uma das demais áreas citadas) -; e também junto a dez trabalhadores sanitaristas lotados na Secretaria Estadual de Saúde de Pernambuco. Solicitou-se de cada um que escolhesse uma intervenção na qual atuava e realizasse sua avaliação, relativamente a cada indicador do instrumento, tendo por referência uma escala de valores de 1 (definitivamente, não atendia ao indicador) a 4 (definitivamente, atendia ao indicador); e fizesse comentários, críticas e sugestões sobre sua clareza especialmente. Após a avaliação individual dos trabalhadores, coordenou-se uma roda de conversa em que mais sugestões foram apresentadas, sendo possível verificar a compreensão do público-alvo sobre a ideia central do instrumento e, assim, confirmar sua utilidade na avaliação das intervenções em saúde. A partir das sugestões apontadas, modificações na versão inicial foram realizadas.

\section{Etapa 3}

Validação de conteúdo - análise do instrumento por especialistas da área de Promoção da Saúde quanto à adequação e clareza dos princípios e dos respectivos indicadores

Em um primeiro momento, foram convidados dez especialistas em Promoção da Saúde, todos professores 
universitários com doutorado e produção na área (três com formação inicial em odontologia, dois em educação física, dois em enfermagem, dois em medicina e um em psicologia), aos quais foi encaminhado 0 instrumento por correio eletrônico, sobre planilha do googleforms@. Alguns desses especialistas foram escolhidos por conveniência; outros, selecionados mediante busca por autores em revistas da área da Saúde Coletiva, a partir do descritor 'Promoção da Saúde'.

No texto introdutório enviado aos especialistas, buscou-se esclarecer que o público-alvo do instrumento são os trabalhadores atuantes em intervenções que buscam promover saúde e que, ao responderem ao instrumento, deveriam selecionar uma intervenção específica (por exemplo: consulta compartilhada, grupo de atividades físicas/práticas corporais, grupo de educação alimentar, grupo de tabagistas, visita domiciliar etc.) para ser avaliada em relação ao nível de proximidade que apresentasse com os indicadores do instrumento. Logo, foi solicitado a esses especialistas avaliarem:

a) se 0 princípio era adequado/pertinente à promoção da saúde;

b) se 0 indicador era adequado/pertinente ao princípio; $\mathrm{e}$

c) se 0 (s) indicador(es) era(m) suficiente(s) para se avaliar o princípio.

Em relação aos itens ' $a$ ' e 'b', a escala de valor considerada foi de 1 (muito inadequado) a 4 (muito adequado), e quanto ao item 'c', de 1 (muito insuficiente) a 4 (muito suficiente). Também havia espaço no instrumento para os especialistas apresentarem sugestões de redação, comentários e críticas sobre o(s) princípio(s) e seu(s) respectivo(s) indicador(es).

Os resultados da parte quantitativa, assim como os comentários dos especialistas, orientaram duas decisões principais: manter apenas os princípios que constam na PNPS $;{ }^{10}$ e apresentar apenas um indicador para cada princípio. Estas mudanças objetivaram deixar o instrumento mais enxuto e direto, de modo a ser mais bem compreendido pelo público-alvo e ter maior aplicabilidade. Dessa forma, uma nova versão do instrumento foi elaborada.

Considerando-se as mudanças realizadas no instrumento, optou-se pela realização de uma nova consulta aos especialistas. Todos os dez especialistas que participaram da etapa anterior foram novamente convidados. Uma vez que somente oito responderam, decidiu-se pelo convite de mais quatro especialistas, totalizando os 12 (três com formação inicial em odontologia, cinco em educação física, dois em enfermagem e dois em medicina) que avaliaram a nova versão. Esses especialistas atuavam em oito diferentes estados brasileiros, Amazonas, Paraíba, Paraná, Pernambuco, Rio de Janeiro - Rio Grande do Sul, Santa Catarina e São Paulo -, além do Distrito Federal.

\section{Etapa 4}

\section{Validação aparente (face) - aplicação com público-alvo (trabalhadores que atuam em intervenções em saúde) da versão final do instrumento}

A versão final do instrumento foi aplicada para 19 residentes da Saúde, especificamente das residências multiprofissionais em Saúde Coletiva e Saúde da Família, da Secretaria de Saúde do Recife. Os residentes tinham diferentes formações: biologia $(n=9)$, educação física $(n=2)$, enfermagem $(n=5)$, farmácia $(n=7)$, fisioterapia $(\mathrm{n}=1)$, fonoaudiologia $(\mathrm{n}=1)$, medicina veterinária $(n=10)$, nutrição $(n=6)$, odontologia $(n=8)$, serviço social $(\mathrm{n}=3)$ e terapia ocupacional $(\mathrm{n}=3)$.

Nessa etapa, inicialmente, solicitou-se de cada participante que avaliasse individualmente uma intervenção da qual participava. Em um segundo momento, os participantes foram divididos em grupos de três a cinco pessoas, sendo-lhes solicitado escolher uma intervenção (todos os membros do grupo deveriam atuar nessa intervenção) e discutir seu nível de aproximação com cada indicador apresentado. Como os indicadores são apresentados de forma assertiva, os residentes/trabalhadores deveriam avaliar cada um segundo a escala de 1 (discordo totalmente) a 4 (concordo totalmente).

Também foi solicitado a cada grupo apresentar os motivos de cada resposta e discutir ações/estratégias possíveis de serem realizadas, para a melhoria dos indicadores que obtiveram avaliações mais negativas. $\mathrm{Na}$ parte final desta etapa, realizou-se uma roda de conversa pautada em um roteiro de duas perguntas:

o instrumento foi útil para identificação de pontos mais "distantes" e mais "próximos" aos princípios da Promoção da Saúde, na intervenção avaliada? 
O instrumento foi útil para promover a definição de estratégias que melhorem a intervenção e aproximemna mais dos princípios da Promoção da Saúde?

Todas as falas foram gravadas e posteriormente transcritas, para análise.

\section{Etapa 5}

\section{Fidedignidade - análise da consistência interna e estabilidade temporal na aplicação da réplica (teste-reteste) com o público-alvo (trabalhadores que atuam em intervenções em saúde) da versão final do instrumento}

A fidedignidade do instrumento foi investigada pela análise da consistência interna (alpha $[\alpha]$ de Cronbach) e estabilidade temporal (concordância entre duas aplicações - teste-reteste). Para tanto, participaram 31 profissionais de saúde atuantes em Florianópolis, Londrina e Recife, das seguintes áreas: educação física $(n=10)$, enfermagem $(n=6)$, farmácia $(n=1)$, fisioterapia $(n=1)$, fonoaudiologia $(n=1)$, medicina veterinária $(\mathrm{n}=2)$, nutrição $(\mathrm{n}=1)$, odontologia $(n=3)$, serviço social $(n=3)$, terapia ocupacional $(n=1)$ e psicologia $(n=2)$. Nesta etapa, solicitou-se a cada participante avaliar individualmente uma intervenção da qual havia participado nos últimos 12 meses. Esta avaliação foi realizada em dois momentos, com intervalo não superior a duas semanas. Reforçou-se que essa avaliação, nos dois momentos, tivesse por objeto a ação/intervenção.

0s dados coletados foram digitados em planilha do aplicativo Microsoft Excel $^{\circledR}$ e analisados com distribuição de frequência absoluta e relativa. A análise da validade de conteúdo e de face adotou a estimativa do índice de validade de conteúdo (IVC), para adequação e clareza dos indicadores do instrumento. 0 IVC mede a proporção de juízes que concordam sobre os indicadores componentes do instrumento. A fidedignidade foi testada por meio da análise da consistência interna e estabilidade temporal. A consistência interna foi verificada a partir do valor de alfa de Cronbach, ${ }^{23}$ que estima a contribuição de cada indicador na composição do escore final. A estabilidade temporal foi verificada pelo método do teste-reteste (intervalo entre sete e 13 dias), e testada por meio do coeficiente de correlação intraclasse (CCI) e da concordância relativa. Para estimativa do CCI, utilizou-se a soma do total dos escores do instrumento. A concordância relativa mostra a concordância entre as respostas nas duas aplicações. Foram considerados valores adequados de validade e fidedignidade: IVC $\geq 70,0 \%$; alfa de Cronbach $\geq 0,70$; CCI $\geq 0,70$; e concordância relativa $\geq 70,0 \%$. As análises foram realizadas pelo software Statistical Package for the Social Sciences (SPSS) versão 21.0, com nível de significância de 5\%.

0 projeto do estudo foi aprovado pelo Comitê de Ética em Pesquisa Envolvendo Seres Humanos da Universidade Estadual de Londrina (CEP/UEL): Parecer $\mathrm{n}^{0}$ 2.855.332, emitido em 30 de agosto de 2018; Certificado de Apresentação para Apreciação Ética (CAAE) $\mathrm{n}^{0}$ 95467618.6.0000.5231.

\section{Resultados}

A Tabela 1 apresenta os resultados relativos à validade de conteúdo, quanto à adequação dos indicadores aos respectivos princípios, e à clareza dos indicadores, na versão final do instrumento. A análise dos especialistas apontou que os indicadores foram adequados para os princípios e se mostraram claros em sua redação. Sobre a adequação dos indicadores aos princípios, a média dos nove indicadores foi de 89,8\%, com todos os indicadores a apresentar escores de concordância maiores que $80,0 \%$ (cinco deles foram maiores que $90,0 \%)$. Quanto à clareza dos indicadores, o valor médio geral foi de $82,5 \%$, variando entre 66,7 e $100,0 \%$; apenas 'empoderamento' teve 0 valor atribuído à clareza desse indicador menor que $70,0 \%$, entre os especialistas. A análise da consistência interna (alfa de Cronbach) demonstrou valor significativo $(\alpha=0,80)$ para os indicadores do instrumento; e aqueles que demostraram importância equivalente e satisfatória para compor o valor total da escala foram mantidos na estrutura final. Os indicadores apresentaram concordância elevada, entre 77,4 e 93,6\%. 0 valor do CCI do instrumento foi de $0,93\left(\mathrm{IC}_{95 \%} 0,85 ; 0,96\right)$.

Em relação à validade de face, destaca-se que todos os trabalhadores consideraram 0 instrumento (Figura 2) útil à identificação de pontos mais "distantes" e "próximos" da intervenção avaliada, no que diz respeito aos princípios da Promoção da Saúde, bem como ao fomento da discussão sobre possíveis estratégias de como melhorar as intervenções, para sua maior aproximação com os princípios da Promoção da Saúde. 
Tabela 1 - Avaliação dos especialistas em relação à adequação e clareza dos indicadores e fidedignidade de um instrumento para avaliar o nível de proximidade de intervenções em saúde com os princípios da Promoção da Saúde

\begin{tabular}{|c|c|c|c|c|}
\hline \multirow[t]{2}{*}{ Princípio } & \multirow[t]{2}{*}{ Indicador } & \multicolumn{2}{|c|}{$\begin{array}{c}\text { Índice de validade } \\
\text { de conteúdo }\end{array}$} & \multirow{2}{*}{$\begin{array}{c}\text { Índice de } \\
\text { fidedignidade } \\
\text { Teste-reteste }^{c}\end{array}$} \\
\hline & & Adequaçãoa & Clareza $^{b}$ & \\
\hline Equidade & $\begin{array}{l}\text { A intervenção atende, de forma diferenciada, indivíduos ou } \\
\text { grupos que mais precisam dela }\end{array}$ & 83,3 & 83,3 & 90,3 \\
\hline Participação social & $\begin{array}{l}\text { A intervenção estimula a participação social dos indivíduos e } \\
\text { grupos }\end{array}$ & 83,3 & 75,0 & 93,6 \\
\hline Autonomia & $\begin{array}{l}\text { A intervenção estimula o desenvolvimento de habilidades } \\
\text { pessoais de seus participantes, buscando que eles sejam mais } \\
\text { autônomos, conscientes e críticos no seu dia a dia, em relação } \\
\text { aos modos/estilos de vida }\end{array}$ & 91,7 & 83,3 & 90,3 \\
\hline Empoderamento & $\begin{array}{l}\text { A intervenção estimula nos participantes o controle das } \\
\text { decisões e das escolhas de modos/estilos de vida diante } \\
\text { das oportunidades, com base em suas condições sociais, } \\
\text { econômicas e culturais }\end{array}$ & 83,3 & 66,7 & 77,4 \\
\hline Intrassetorialidade & $\begin{array}{l}\text { A intervenção estimula a articulação entre atores de um } \\
\text { mesmo setor, buscando a construção e articulação de redes } \\
\text { cooperativas e resolutivas }\end{array}$ & 91,7 & 91,7 & 80,7 \\
\hline Intersetorialidade & $\begin{array}{l}\text { A intervenção estimula a articulação entre diferentes setores, } \\
\text { buscando a construção de intervençỗes compartilhadas, } \\
\text { pautadas na corresponsabilidade e cogestão }\end{array}$ & 91,7 & 75,0 & 90,3 \\
\hline Sustentabilidade & $\begin{array}{l}\text { A intervenção oferece condições para continuidade, enquanto } \\
\text { for prioritária para o território }\end{array}$ & 100,0 & 91,7 & 80,7 \\
\hline Integralidade & $\begin{array}{l}\text { A intervenção considera a complexidade, potencialidade e } \\
\text { singularidades de seus participantes }\end{array}$ & 83,3 & 75,0 & 77,4 \\
\hline Territorialidade & $\begin{array}{l}\text { A intervenção considera as especificidades do território onde } \\
\text { está inserida }\end{array}$ & 100,0 & 100,0 & 80,6 \\
\hline Média geral & & 89,8 & 82,5 & 84,6 \\
\hline
\end{tabular}

a) Percentual de especialistas que consideraram o indicador muito adequado ou adequado ao princípio; b) Percentual de especialistas que consideraram o indicador muito claro ou claro; c) Percentual de especialistas que concordaram com as respostas nas réplicas de aplicação (teste-reteste).

\section{Discussão}

Os resultados obtidos apontam que o instrumento proposto apresentou validade de conteúdo e face, fidedignidade, todas essas qualidades aceitáveis quanto a sua estrutura, podendo ser utilizado por trabalhadores atuantes em intervenções destinadas a promover saúde e, especificamente, avaliar a adequação dessas intervenções aos princípios da PNPS. Apesar de, inicialmente, ter sido pensado para trabalhadores da Atenção Primária à Saúde, e admitindo-se a probabilidade de esses trabalhadores utilizarem-no com mais frequência, verificou-se que o instrumento poderia ser adotado por profissionais de outros setores, conforme destacado pelos especialistas consultados.

Entretanto, algumas limitações necessitam ser consideradas. A inclusão de outros indicadores, certamente, aumentaria a variabilidade e complexidade já observada em muitos princípios do instrumento - ainda que esse incremento pudesse reduzir sua aplicabilidade. Porém, mesmo com um indicador em cada princípio, a análise de validade e fidedignidade apontou resultados adequados, com valores psicométricos aceitáveis. Vale destacar que dificilmente uma intervenção alcançará a perfeição em todos os indicadores. Talvez uma avaliação perfeita, ou perto disso, mais reflita uma falta de atuocrítica do avaliador do que propriamente a qualidade da intervenção. Além disso, é possível que, para determinadas intervenções, um ou mais indicadores não se apliquem. Também é importante destacar que a avaliação negativa de determinado indicador não necessariamente representa "falha" na atuação do trabalhador que faz a avaliação. Por exemplo, na aplicação-piloto da versão inicial do 


\section{INSTRUMENTO PARA AVALIAÇÃO DAS INTERVENÇÕES EM SAÚDE QUANTO À APROXIMAÇÃO COM OS PRINCÍPIOS DE PROMOÇÃO DA SAÚDE}

O OBJETIVO DESTE INSTRUMENTO É AUXILIAR OS TRABALHADORES A AVALIAREM AS INTERVENÇÕES QUE TÊM COMO FOCO A PROMOÇÃO DA SAÚdE, FACILITANDO A IDENTIFICAÇÃO DE POTENCIALIDADES E FRAGILIDADES DESTAS INTERVENÇōES, QUE DEVEM BUSCAR A MAIOR APROXIMAÇÃO POSSÍVEL COM OS PRINCÍPIOS DA POLÍTICA NACIONAL DE PROMOÇÃO DA SAÚDE (PNPS).

O INSTRUMENTO PODE SER RESPONDIDO INDIVIDUALMENTE OU POR UM GRUPO DE TRABALHADORES QUE ATUAM NA INTERVENÇ̃̃O. NESTE CASO, SUGERE-SE QUE DISCUTAM CADA ITEM E BUSQUEM CHEGAR A CONSENSOS OU REALIZEM ALGUMA MEDIDA A PARTIR DA MÉEIA DAS AVALIAÇ̄̃ES INDIVIDUAIS.

PARA RESPONDER AO INSTRUMENTO, DEVEM-SE CONSIDERAR OS SEGUINTES CONCEITOS OPERACIONAIS:

PROMOÇÃO DA SAÚdE - ÉUM CONJUNTO DE ESTRATÉGIAS E FORMAS DE PRODUZIR SAÚDE, NO ÂMBITO INDIVIDUAL E COLETIVO, QUE SE CARACTERIZA PELA ARTICULAÇ̃̃O E COOPERACÃ̃O INTRASSETORIAL E INTERSETORIAL E PELA FORMAÇÃO DA REDE DE ATENÇÃo À SAÚdE, BUSCANDO SE ARTIICULAR COM AS DEMAIS REDES DE PROTEÇÃO SOCIAL, COM AMPLA PARTIIIPAÇ̧̃̈O E CONTROLE SOCIAL (BRASIL, 2015).

INTERVENÇ̄̃O - QUALQUER ATIVIDADE SISTEMATIZADA, REALIZADA POR UM OU MAISTRABALHADORES, OUE OBJETIVEM MUDANCAS POSITIVAS EM SAÚDE EM INDIVÍDUOS E/OU GRUPOS. EXEMPLOS: UM GRUPO DE EDUCAÇÃO EM SAÚDE, GRUPO DE PRÁTICAS CORPORAIS/ATIVIDADE FÍSICA, ATENDIMENTO COMPARTILHADO, VIIITA DOMICILIAR ETC.

\begin{tabular}{|l|l|}
\hline LOCAL DA INTERVENÇÃO & \\
\hline INTERVENÇÃo & \\
\hline
\end{tabular}

AGORA AVALIE(M) A INTERVENÇ̃̃ ESCOLHIDA, MARCANDO UM "X" NO NÚMERO QUE MELHOR REPRESENTA O QUANTO VOCÊ(S) ESTÁ(0) DE ACORDO COM AS AFIRMAÇÕES DOS ITENS ABAIXO, CONSIDERANDO A SEGUINTE ESCALA:

\begin{tabular}{|c|c|c|c|}
\hline 1 & 2 & 3 & 4 \\
\hline DISCORDO & CONCORDO POUCO & CONCORDO & CONCORDO MUITO \\
\hline
\end{tabular}

\begin{tabular}{|c|c|c|}
\hline $\mathbf{N}^{0}$ & PRINCÍPIO & ITEM \\
\hline 1 & EQUIDADE & $\begin{array}{l}\text { A INTERVENÇÃO ATENDE DE FORMA DIFERENCIADA INDIVÍDUOS OU GRUPOS QUE MAIS PRECISAM } \\
\text { DA MESMA }\end{array}$ \\
\hline 2 & PARTICIPAÇÃOSOCIAL & A INTERVENÇÃO ESTIMULA A PARTIIIPAÇÃO SOCIAL DOS INDIVÍDUOS E GRUPOS \\
\hline 3 & AUTONOMIA & $\begin{array}{l}\text { A INTERVENÇÃO ESTIMULA O DESENVOLVIMENTO DE HABILIDADES PESSOAIS DE SEUS PARTICIPANTES, } \\
\text { BUSCANDO QUE ESTES SEJAM MAIS AUTOONOMOS, CONSCIENTES E CRÍTICOS NO SEU DIA A DIA EM } \\
\text { RELAÇÃO AOS MODOS/ESTILOS DE VIDA }\end{array}$ \\
\hline 4 & EMPODERAMENTO & $\begin{array}{l}\text { A INTERVENÇÃO ESTIMULA NOS PARTICIPANTES O CONTROLE DAS DECISÕES E DAS ESCOLHAS DE } \\
\text { MODOS/ESTILOS DEVIDA DIANTE DAS OPORTUNIDADES, CONSIDERANDO AS SUAS CONDIÇÕES SOCIAIS, } \\
\text { ECONÔMICAS E CULTURAIS }\end{array}$ \\
\hline 5 & INTRASSETORIALIDADE & $\begin{array}{l}\text { A INTERVENÇÃO ESTIMULA A ARTICULAÇÃO ENTRE ATORES DE UM MESMO SETOR, BUSCANDO A } \\
\text { CONSTRUÇÃO E ARTICULAÇÃO DE REDES COOPERATIVAS E RESOLUTIVAS }\end{array}$ \\
\hline 6 & INTERSETORIALIDADE & $\begin{array}{l}\text { A INTERVENÇÃO ESTIMULA A ARTICULAÇÃ0 ENTRE DIFERENTES SETORES, BUSCANDO A CONSTRUÇÃO } \\
\text { DE INTERVENÇÕES COMPARTILHADAS, ENVOLVENDO CORRESPONSABILIDADE E COGESTÃO }\end{array}$ \\
\hline 7 & SUSTENTABILIDADE & $\begin{array}{l}\text { A INTERVENÇÃO OFERECE CONDIÇ̃̃ES PARA QUE TENHA CONTINUIDADE ENQUANTO FOR PRIORITÁRIA } \\
\text { PARA O TERRITÓRIO }\end{array}$ \\
\hline 8 & INTEGRALIDADE & $\begin{array}{l}\text { A INTERVENÇÃO CONSIDERA A COMPLEXIDADE, POTENCIALIDADE E SINGULARIDADES DE SEUS } \\
\text { PARTICIPANTES }\end{array}$ \\
\hline 9 & TERRITORIALIDADE & A INTERVENÇÃO CONSIDERA AS ESPECIFICIDADES DO TERRITÓRIO ONDE ESTÁ INSERIDA \\
\hline
\end{tabular}

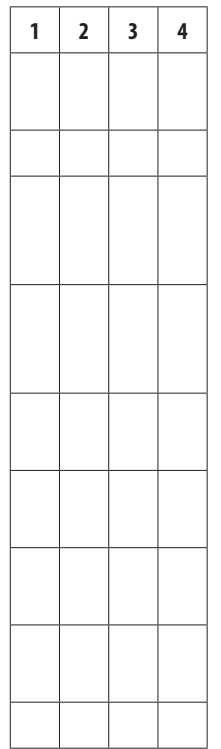

ADICIONALMENTE, SUGERE-SE QUE OS RESPONDENTES APONTEM EM CADA ITEM OS MOTIVOS PARA O CONCEITO ATRIBUÍDO (NÚMERO/ ESCALA DE 1 A 4) E PENSEM ESTRATÉGIAS VIÁVEIS PARA MELHORIA DOS ITENS COM AVALIAÇÃO MAIS BAIXA.

Figura 2 - Instrumento para avaliação de intervenções em saúde quanto a sua aproximação com os princípios da Promoção da Saúde 
instrumento, 0 indicador relacionado à intersetorialidade não foi bem avaliado em uma das intervenções. $\mathrm{Na}$ discussão, ficou evidenciado que a responsabilidade não era somente dos envolvidos na intervenção sob avaliação. Entretanto, percebeu-se a necessidade de buscar uma melhor articulação com outros setores.

0 instrumento pode auxiliar no preenchimento de uma lacuna, relacionada à escassez de outras formas de avaliação das ações focadas na Promoção da Saúde. Ele pode ajudar os trabalhadores a elaborar adequações em alguns aspectos de suas intervenções, ou passar a enfatizar aspectos não considerados até então. Por exemplo: os responsáveis por uma determinada intervenção percebem que essa intervenção não tem estimulado a participação social. Certamente, uma ação relativamente simples e viável seria a divulgação das datas das reuniões dos Conselhos Municipais de Saúde, inclusive dos conselhos de moradores do bairro, com a devida ênfase à importância desses organismos para a efetiva Participação Social, um dos princípios do Sistema Único de Saúde (SUS).

Sobre a operacionalização da Promoção da Saúde no cotidiano dos serviços, vale ressaltar a forma bastante diversa como o conceito se traduz em ações concretas, ${ }^{24}$ razão por que se deve reforçar a importância do processo de avaliação das ações em saúde; e, considerando-se a Promoção da Saúde em particular, sua abordagem de ordem teórica e da práxis, como componente do cuidado integral. Portanto, cresce a necessidade de definir ferramentas que permitam avaliar essas práticas e sejam úteis à orientação desse processo, tanto no nível da micro como da macropolítica. Nesse cenário, o uso do presente instrumento poderá conduzir a uma intencionalidade da construção/planejamento e avaliação das ações de Promoção de Saúde.

Um ponto importante a ser destacado, observado nas etapas em que os trabalhadores apresentaram suas percepções sobre a possível utilidade do instrumento (etapas 2 e 4), diz respeito a sua possível contribuição para que se supere a noção de uma intervenção em saúde "curativa" ou "promotora de saúde" tão somente. A definição de uma ação como "promotora de saúde" não deve ser "apriorística", o que muitas vezes acontece quando determinadas temáticas e ações são classificadas como promotoras de saúde e outras não, sem a devida análise da forma como são efetivadas. ${ }^{25}$ Nesta mesma linha de raciocínio, a Promoção da Saúde não está apenas nos fins, mas também, fundamentalmente, nos meios, inclusive na análise de como as ações são planejadas e executadas. ${ }^{26}$

0 instrumento apresentou validação de conteúdo e de face, análise de fidedignidade (consistência interna e estabilidade temporal) aceitável, e reúne potencial para ser utilizado pelos trabalhadores envolvidos em intervenções cujo objetivo é promover saúde. Especialmente, as intervenções de Promoção da Saúde devem ser avaliadas em sua aproximação com os princípios da PNPS. Naturalmente, a especificidade de cada contexto e intervenção deve ser considerada, uma vez que a maior aproximação depende de diversos fatores, entre eles as próprias condições de trabalho e a disposição dos trabalhadores para uma avaliação franca de suas intervenções.

\section{Contribuição dos autores}

Loch MR e Lemos EC contribuíram na concepção e delineamento do estudo, análise e interpretação dos resultados, redação e revisão crítica do conteúdo do manuscrito. Jaime PC e Rech CR contribuíram na análise e interpretação dos resultados, revisão crítica do conteúdo e interpretação dos resultados, redação e revisão crítica do conteúdo do manuscrito. Todos os autores aprovaram a versão final do manuscrito e são responsáveis por todos os seus aspectos, incluindo a garantia de sua precisão e integridade. 


\section{Referências}

1. Sicoli JL, Nascimento PR. Promoção de saúde: concepções, princípios e operacionalização. Interface (Botucatu). 2003;7(12):101-22. doi: http://doi. org/10.1590/S1414-32832003000100008.

2. Heidmann ITSB, Almeida MCP, Boehs AE, Wosny AM, Monticelli M. Promoção à saúde: trajetória histórica de suas concepções. Texto contextoEnferm. 2006;15(2): 352-8. doi: http://doi. org/10.1590/S0104-07072006000200021.

3. Czeresnia D, Freitas CM, organizadores. Promoção da saúde: conceitos, reflexões, tendências. Rio de Janeiro: Editora Fiocruz; 2009.

4. Westphal MF. Promoção da saúde e prevenção de doenças. In: Campos GWS, Minayo MCS, Akerman N, Drumond Júnior M, Carvalho YM, organizadores. Tratado de saúde coletiva. 2a ed. São Paulo: Hucitec; 2014. p. 681-717.

5. Bezerra IMP, Sorpreso ICE. Concepts and movements in health promotion to guide educational practices. J Hum Growth Dev. 2016;26(1):11-16. doi: http://doi.org/10.7322/jhgd.113709.

6. Mendes R, Fernandez JCA, Sacardo DP. Promoção da saúde e participação: abordagens e indagações. Saude Debate. 2016; 40(108):190-203. doi: http:// doi.org/10.1590/0103-1104-20161080016.

7. Souza RKT, Loch MR. Promoção da saúde. In: Andrade SM, Cordoni Júnior L, Carvalho BG, Gonzáles AD, Silva AMR, organizadores. Bases da saúde coletiva. $2^{\text {a }}$ ed. Londrina: 2017. p. 29-46.

8. Colin ECS, Pelicioni CF. Territorialidade, desenvolvimento local e promoção da saúde: estudo de caso em uma vila histórica de Santo André, São Paulo. Saude Soc. 2018;27(4):1246-60. doi: http://doi.org/10.1590/s0104-12902018170850.

9. Chiari APG, Ferreira RC, Akerman M, Amaral JHL, Machado KM, Senna MIB. Rede intersetorial do Programa Saúde na Escola: sujeitos, percepções e práticas. Cad. Saude Publica. 2018.34(5):e00104217: 2-14. doi: http://doi.org/10.1590/0102-311x00104217.

10. Brasil. Portaria n. 2446, de 11 de novembro de 2014. Redefine a Política Nacional de Promoção da saúde (PNPS). Brasília, DF: Diário Oficial da União. 13 nov. 2014 [acesso 18 jun. 2019]: Seção 1, p. 68. Disponível em: http://bvsms.saude.gov.br/bvs/ saudelegis/gm/2014/prt2446_11_11_2014.html.
11. Malta DC, Reis CAA, Jaime PC, Moraes Neto OL, Silva MMA, Akerman Met. 0 SUS e a política nacional de promoção da saúde: perspectiva resultados, avanços e desafios em tempos de crise. Cienc Saude Colet. 2018;23(6):1799-809. doi: http://doi. org/10.1590/1413-81232018236.04782018.

12. Rocha DG, Alexandre VP, Marcelo VC, Rezende R, Nogueira JD, Sá RF. Processo de revisão da política nacional de promoção da saúde: múltiplos movimentos simultâneos. Cienc Saude Colet. 2014;19(11):4313-22. doi: http://doi. org/10.1590/1413-812320141911.11232014.

13. Malta DC, Morais Neto OL, Silva MMA, Rocha D, Castro AM, Reis AAC, et al. Política nacional de promoção da saúde (PNPS): capítulos de uma caminhada ainda em construção. Cienc Saude Colet. 201621(6):1683-94. doi: http://doi.org/10.1590/1413-81232015216.07572016.

14. DeVellis RF. Scale development: theory and applications, 4th ed. publisher unknown: SAGE: 2017.

15. World Health Organization. The Ottawa charter for health promotion: the first international conference on health promotion, 1986 [acesso 7 fev 2020] Geneva: WH0; [1987?]. Disponível em: https:/www.who.int/ healthpromotion/conferences/previous/ottawa/en/

16. World Health Organization. Adelaide recommendations on healthy public policy 1988. [acesso 7 fev. 2020] Disponível em: http:// www.who.int/hpr/docs/adelaide.html

17. World Health Organization. Sundsvall Statement on supportive environments for health 1991 [ acesso 7 fev. 2020] Disponível em: https://archive. org/details/WHOHED92.1/page/n2/mode/2up

18. World Health Organization. Jakarta declaration on leading health promotion into the 21st Century 1997. Rev Salud Publica. 1998 [acesso 7 fev. 2020]];3(1):58-61. Disponível em: https:// scielosp.org/pdf/rpsp/1998.v3n1/58-61/en

19. [Organização Mundial da Saúde].. Declaração do México promoção da saúde: rumo à maior equidade, 2000 [acesso 7 fev. 2020]. Disponível em: http://bvsms.saude.gov.br/bvs/ publicacoes/declaracao_mexico_2000.pdf

20. World Health Organization. The Bangkok charter for health promotion in a globalized world 2005 [acesso 7 fev. 2020]. Disponível em: http://www.iasaude.pt/ attachments/article/66/Carta_de_BangKoK-2005.pdf 
21. World Health Organization. Nairobi call to action, 2009. Geneva: WHO; 2009 [acesso 7 fev. 2020] Disponível em: http://www.ngos4healthpromotion. net/wordpressa4hp/wp-content/uploads/2017/04/ Nairobi_Call_to_Action_Nov09.pdf

22. World Health Organization. The Helsinki statement on health in all policies: the 8th global conference on health promotion, 2013. Geneva: WHO; 2013 [acesso 7 fev. 2020]. Disponível em: http://www. who.int/healthpromotion/conferences/8gchp/en/.

23. Souza AC, Alexandre NMC, Guirardello EB. Psychometric properties in instruments evaluation of reliability and validity. Epidemiol Serv Saude. 2017;26(3):647-57. doi: http://doi. org/10.5123/S1679-49742017000300022.
24. Medina MG, Aquino R, Vilasbôas ALQ, Mota E, Pinto Júnior EP, Luz LA, et al. Promoção da saúde e prevenção de doenças crônicas: o que fazem as equipes de Saúde da Família? Saude Debate. 2014;38(n.spe):6982. doi: http://doi.org/10.5935/0103-1104.2014S006.

25. Carvalho FFB, Cohen SC, Akerman M. Promoção da saúde na atenção básica: problematizar o instituído para aproximá-la do cuidado. Porto Alegre (RS): Artmed Panamericana; 2018. p. 9-37. (Sistema de Educação Continuada a Distância, vol. 3).

26. Carvalho FFB. A Promoção da saúde e a superação do instituído: caminhos para a aproximação com o cuidado [tese]. Rio de Janeiro: Fundação Oswaldo Cruz; 2019.

\section{Abstract}

objective: To develop and validate of an instrument to evaluate interventions in relation to Health Promotion principles in Brazil. Methods: A cross-sectional study of the development and validation of an instrument was carried out, based on the assessment of the first version by 20 bealth workers, the final version by 19, analysis of reliability by 31, and content evaluation of the first version by ten experts and of the final version by 12 experts. The content validity index, Cronbach's alpha ( $\alpha$ ) and intraclass correlation coefficient were used. Results: The indicators achieved $89.9 \%$ adequacy, 82.5\% clarity, internal consistency was $\alpha=0.80$ and the test-retest correlation was 0.93. Conclusion: The instrument showed acceptable validity and reliability and can be used for the evaluation of Health Promotion interventions.

Keywords: Health Promotion; Validity Studies; Surveys and Questionnaires; Health Care Models.

\section{Resumen}

Objetivo: Desarrollar y validar un instrumento para evaluar la proximidad de intervenciones que buscan promover la salud con los principios de la Política Nacional de Promoción de la Salud de Brasil. Métodos: Se realizó estudio transversal del desarrollo y validación de un instrumento, a partir del análisis de 20 trabajadores en el análisis de la primera versión, 19 en la versión final, 31 para el análisis de confiabilidad y la evaluación de contenido por 10 especialistas en la versión inicial y 12 en la versión final. En el análisis se utilizaron el indice de validez de contenido, el alfa de Cronbach ( $\alpha$ ) y el coeficiente de correlación intraclase. Resultados: La adecuación de los indicadores fue del $89,8 \%$, la claridad del 82,5\%, la consistencia interna de $\alpha=0,80$ y la correlación test-retest 0,93. Conclusión: $E l$ instrumento mostró validez y confiabilidad aceptables y se puede utilizar para evaluar intervenciones que buscan promover la salud.

Palabras clave: Promoción de la Salud; Estudio de Validación; Encuestas y Cuestionarios; Modelos de Atención de Salud.

Recebido em 03/09/2020

Aprovado em 21/01/2021

Editora associada: Lúcia Rolim Santana de Freitas - (1) orcid.org/0000-0003-0080-2858 Editora científica: Taís Freire Galvão - (D) orcid.org/0000-0003-2072-4834 Editora geral: Leila Posenato Garcia - (1) orcid.org/0000-0003-1146-2641 